\title{
Toll-like receptor expression in severe asthma with chronic rhinosinusitis
}

\author{
Carina Malmhällı, Apostolos Bossios, Margareta Sjöstrand, Bo Lundbäck, Jan Lötvall, Madeleine Rådinger \\ From EAACI International Severe Asthma Forum (ISAF 2012) \\ Gothenburg, Sweden. 11-13 October 2012
}

\begin{abstract}
Background
We have in an epidemiological study identified a group of individuals with multiple asthma symptoms (MSA) that reflect a more severe and uncontrolled disease. An altered innate immunity and adaptive immunity, and effects of microorganisms may play a role in the development of both CRS and asthma. The innate immune system use Toll-like receptors (TLRs) to recognize microbes and activate defense mechanisms within minutes of microbial invasion which is followed by an antigen-specific response by the adaptive immune system. Thus, innate and adaptive immune cells are sequentially activated and reciprocally regulate one another. Glycogen synthase kinase-3 $\beta$ (GSK3 $\beta$ ) has been demonstrated as a regulator of both innate and adaptive immunity. GSK3 $\beta$ was shown to modulate inflammatory responses through TLR-mediated production of pro-inflammatory cytokines and inactivation of GSK3 $\beta$ (phosphorylated GSK3 $\beta$ ) lead to a reduced IL-12 production, which was suggested to skew the balance towards a Th2 response.
\end{abstract}

\section{Aim}

To determine the degree of expression of TLRs 2, 4, 7 and 9 on monocytes as well phGSK3 $\beta$ using flow cytometry. We hypothesize that the co-existence of CRS in patients with asthma and especially patients with MSA has an increased TLR expression involved in the innate immune system.

\section{Method}

Participants were selected from an epidemiological cohort, the West Sweden Asthma Study. Clinical parameters as well as fresh peripheral blood cells were obtained from one group of non-asthmatic subjects with CRS (CRS) and four different groups of asthmatics: 7 subjects with MSA
(MSA), 7 subjects with other asthma (OA), 7 subjects with MSA and CRS (MSA/CRS) and 9 subjects with OA and CRS (OA/CRS). These five groups were compared to a control group consisting of 10 healthy subjects without asthma or CRS.

\section{Results}

Individuals with MSA or CRS only, showed significantly increased TLR2, TLR7 and TLR9 expression (rMFI) on $\mathrm{CD} 14^{+}$monocytes compared to controls as well as the expression of phGSK3 $\beta$ and CD14. Individuals in the combined MSA/CRS group showed increased expression of CD14 and TLR2, but increased expression of TLR4 was only found in the CRS group.

\section{Conclusion}

The upregulated expression of TLRs in the MSA group compared to control group suggest a higher susceptibility towards an altered immune response that might reflect the degree of severity. Furthermore, the increased phosphorylation of GSK3 $\beta$ may indicate a switch in the immune response from a pro-inflammatory to a dysregulated Th2 response.

Published: 3 May 2013

doi:10.1186/2045-7022-3-S1-O2

Cite this article as: Malmhäll et al:: Toll-like receptor expression in severe asthma with chronic rhinosinusitis. Clinical and Translational Allergy 2013 3(Suppl 1):O2. 